\title{
Nucleon form factors from high statistics mixed-action calculations with $2+1$ flavors
}

LHPC Collaboration: W. Schroers ${ }^{* a b}$, J.D. Bratt ${ }^{c}$, R.G. Edwards ${ }^{d}$, M. Engelhardt ${ }^{e}$, G.T. Fleming ${ }^{f}$, Ph. Hägler ${ }^{g}$, H.W. Lin ${ }^{d}$, M.F. Lin ${ }^{c}$, H.B. Meyer ${ }^{c}$, B. Musch ${ }^{g}$, J.W. Negele ${ }^{c}$, K. Orginos ${ }^{h}$, A.V. Pochinsky ${ }^{c}$, M. Procura ${ }^{c}$, D.B. Renner ${ }^{i}$, D.G. Richards ${ }^{d}$, S.N. Syritsyn ${ }^{c}$, and A.P. Walker-Loud ${ }^{h}$

${ }^{a}$ Institute of Physics, Academia Sinica, Taipei 115, Taiwan (R.O.C.)

${ }^{b}$ Department of Physics, Center for Theoretical Sciences, National Taiwan University, Taipei 10617, Taiwan (R.O.C.)

${ }^{c}$ Center for Theoretical Physics, Massachusetts Institute of Technology, Cambridge, MA 02139

${ }^{d}$ Thomas Jefferson National Accelerator Facility, Newport News, VA 23606

${ }^{e}$ Department of Physics, New Mexico State University, Las Cruces, NM 88003-0001

${ }^{f}$ Sloane Physics Laboratory, Yale University, New Haven, CT 06520

${ }^{g}$ Institut für Theoretische Physik T39, Physik-Department der TU München, James-Franck-Straße, D-85747 Garching, Germany

${ }^{h}$ Department of Physics, College of William and Mary,P.O. Box 8795, Williamsburg VA 23187-8795

${ }^{i}$ NIC/DESY, D-15738 Zeuthen, Germany

\begin{abstract}
We present new high-statistics results for nucleon form factors at pion masses of approximately $290,350,500$, and $600 \mathrm{MeV}$ using a mixed action of domain wall valence quarks on an improved staggered sea. We perform chiral fits to both vector and axial form factors and compare our results to experiment.
\end{abstract}

The XXVII International Symposium on Lattice Field Theory - LAT2009

July 26-31 2009

Peking University, Beijing, China

* Speaker. 


\section{Introduction}

The proton and the neutron are the fundamental building blocks of our world. They form heavier nuclei and thus the basis for atoms and are the only known source of stable baryonic matter. Their structure can be studied by scattering leptons off nuclei and the most basic observables obtained from these processes are the nucleon form factors. The electromagnetic form factors are Lorentz-scalars which parametrize the matrix element of the electromagnetic current between two nucleon states at different momentum:

$$
\left\langle p^{\prime}\left|\bar{q} \gamma^{\mu} q\right| p\right\rangle=\left\langle\left\langle\gamma^{\mu}\right\rangle\right\rangle F_{1}\left(Q^{2}\right)+\frac{\mathrm{i}}{2 m_{N}}\left\langle\left\langle\sigma^{\mu \alpha}\right\rangle\right\rangle \Delta_{\alpha} F_{2}\left(Q^{2}\right),
$$

where $\left\langle\langle\mathscr{X}\rangle \equiv \bar{u}\left(p^{\prime}\right) \mathscr{X} u(p)\right.$ and $Q^{2} \equiv-\Delta^{2}=-\left(p^{\prime}-p\right)^{2} . m_{N}$ refers to the nucleon mass and the quarks $q$ always refer to the isovector combination $u-d$, i.e. proton minus neutron currents. In this case, contributions from disconnected diagrams cancel due to isospin symmetry. A different parametrization called the Sachs form factors, $G_{E}\left(Q^{2}\right)$ and $G_{M}\left(Q^{2}\right)$, is often used in the literature. We will discuss those in our upcoming paper [1].

In a similar way, the axial current can be parametrized in terms of two form factors. For the isovector current, i.e. the proton minus the neutron combination, they are called the axial form factor, $G_{A}\left(Q^{2}\right)$, and the pseudoscalar form factor, $G_{P}\left(Q^{2}\right)$ :

$$
\left\langle p^{\prime}\left|\bar{q} \gamma_{5} \gamma^{\mu} q\right| p\right\rangle=\left\langle\left\langle\gamma^{\mu} \gamma_{5}\right\rangle\right\rangle G_{A} Q^{2}+\frac{1}{2 m_{N}} \Delta^{\mu}\left\langle\left\langle\gamma_{5}\right\rangle\right\rangle G_{P}\left(Q^{2}\right) .
$$

The current work is based on mixed action calculations using two flavors of dynamical asqtad sea quarks [2] and domain wall valence quarks. In previous years, we have reported on several other nucleon structure observables using this technology, see e.g. [3, 4, 5, 6]. We have studied form factors on full DWF lattices in Ref. [7] which also includes comparison to the work reported here. For a concise review of key results, we refer to Ref. [8]. For recent results from other groups, cf. Ref. [9], and for recent reviews of the field Ref. [10, 11].

The current report focuses on selected results of nucleon form factors and uses several technological updates. The final report using these improvements will be published soon [1] and will include several other major observables like generalized parton distributions and structure functions.

\section{Lattice technology}

As in our previous studies we employ the asqtad action for the sea quarks and the domain wall (DWF) action for the valence quarks. In addition, we also add one lighter mass to our data set. The tuning of the quarks masses and the choice of parameters have been discussed in Ref. [3]. The lattice spacing for all data sets corresponds to $a=0.12406 \mathrm{fm}$ with an uncertainty of $2 \%$, cf. Ref. [12]. This yields a physical volume $V=(2.5 \mathrm{fm})^{3}$ on the $20^{3}$ and $V=(3.5 \mathrm{fm})^{3}$ on the $28^{3}$ lattices. The resulting physical values of the nucleon and pion masses are needed for our calculation and have been determined previously in Ref. [4]. Table 1 lists these numbers.

In previous publications we often computed propagators by chopping each lattice in two halves and performing propagator calculations independently on both halves, cf. Ref. [3] and references 


\begin{tabular}{c|c|c|c|c|c}
\hline \hline Light $\mathbf{m}_{\text {sea }}^{\text {Astad }}$ & Volume & $(\mathbf{a m})_{\pi}$ & $(\mathbf{a m})_{\mathbf{N}}$ & $\mathbf{m}_{\pi} / \mathbf{M e V}$ & $\mathbf{m}_{\mathbf{N}} / \mathbf{M e V}$ \\
\hline 0.007 & $20^{3} \times 64$ & $0.1842(7)$ & $0.696(7)$ & $292.99(111)$ & $1107.1(111)$ \\
0.010 & $28^{3} \times 64$ & $0.2238(5)$ & $0.726(5)$ & $355.98(80)$ & $1154.8(80)$ \\
0.010 & $20^{3} \times 64$ & $0.2238(5)$ & $0.726(5)$ & $355.98(80)$ & $1154.8(80)$ \\
0.020 & $20^{3} \times 64$ & $0.3113(4)$ & $0.810(5)$ & $495.15(64)$ & $1288.4(80)$ \\
0.030 & $20^{3} \times 64$ & $0.3752(5)$ & $0.878(5)$ & $596.79(80)$ & $1396.5(80)$ \\
\hline \hline
\end{tabular}

Table 1: Physical pion and nucleon masses.

therein. In the present work we adopted a different technology and compute multiple source/sink pairs on a single gauge field. We find this approach both more convenient and more powerful, resulting in a superior statistical quality of our results. By choosing eight different source/sink pairs on a single gauge field, we managed to reduce our error bars by a factor of two. We also took possible sources of correlations into account by performing fits using the error correlation matrix among all data points on each ensemble, see e.g. [6], and the "super jackknife" technique, Refs. [13, 14], for combining data from different ensembles in a single fit.

\section{Form factor results}

We discuss several results of our calculation of nucleon form factors. To study the shape of the nucleon at large distances - which is a property that can be studied well by lattice calculations - we perform an expansion of the form factors at small $Q^{2}$, yielding the mean square radii, $\left\langle r_{i}^{2}\right\rangle$, as the slope, where $i$ denotes either 1,2, or $A$, corresponding to the form factor $F_{1}, F_{2}$, or $G_{A}$, respectively. Phenomenologically, the Dirac radius $\left\langle r_{1}^{2}\right\rangle$ can be determined from fits to the form factor $F_{1}\left(Q^{2}\right)$ [15] or from an analysis based on dispersion theory [16, 17, 18]. These two methods currently yield inconsistent results. For $F_{2}\left(Q^{2}\right)$ there is a systematic discrepancy between spintransfer and Rosenbluth experiments, the source is generally believed to be two-photon exchange processes, see Ref. [19] and references therein. A precise quantitative analysis still needs to be done. Lattice calculations allow for a study without two-photon contamination and thus can be very useful in resolving this discrepancy.

We have studied the form factors $F_{1}\left(Q^{2}\right)$ and $F_{2}\left(Q^{2}\right)$ using dipole and tripole fits and also fit our lattice data to the simultaneous expansion in $Q^{2}$ and $m_{\pi}$ obtained from the small-scale expansion (SSE), see Refs. [20, 21] for the explicit form of these expressions. The advantage of the simultaneous expansion is that we do not make model-dependent assumptions on the $Q^{2}$ dependence of the form factors, at variance with the use of dipole or tripole phenomenological formulae. The disadvantage is that the validity of the expansion will only hold for small values of $Q^{2}$ and we have only few data points in that region. Thus, relying on the SSE expansion may increase the uncertainty, both statistical and systematic (which accounts for the unknown magnitude of higher order contributions). Since it is not feasible to determine all the low-energy constants involved in the chiral expressions by fitting to our lattice results, we fix some of them using their phenomenological values.

We find that applying the cuts $Q^{2}<0.5 \mathrm{GeV}^{2}$ and $m_{\pi}<400 \mathrm{MeV}$ yields an acceptable fit with $\chi^{2} /$ dof $=1.86$ with two fit parameters. We could still describe the functional dependence well 
for larger $Q^{2}$, but the functional dependence on $m_{\pi}$ is not too flat in our lattice data compared to the chiral expansion. Despite this observation, we believe that the apparent agreement between the lattice data and the SSE form for $Q^{2}>0.5 \mathrm{GeV}^{2}$ is merely accidental since we have no reason to believe that the SSE at the order given is valid for that range of squared momentum transfer. Figure 1 summarizes our results. The left panel shows the isovector form factor $F_{1}\left(Q^{2}\right)$ lattice data with the best fit SSE at $m_{\pi}=292.99 \mathrm{MeV}$. The right panel shows the resulting chiral extrapolation of the Dirac radii as a function of the pion mass, $m_{\pi}$. For illustration purposes, we have also included the Dirac radii obtained from dipole fits in the graph. However, these data points have no influence on the curve presented and simply compare the two fit methodologies. The red star shows the empirical value taken from Ref. [15].
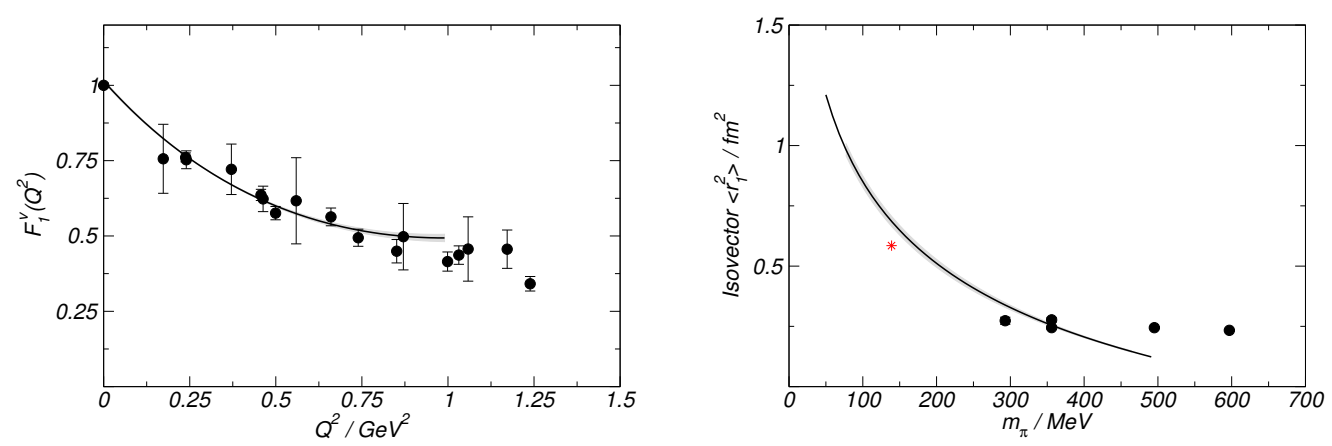

Figure 1: Isovector form factor $F_{1}\left(Q^{2}\right)$ lattice data with best fit SSE at $m_{\pi}=292.99 \mathrm{MeV}$ (left panel) and the resulting isovector Dirac radii, $\left\langle r_{q}^{2}\right\rangle$ (right panel).

The corresponding fit for the isovector $F_{2}\left(Q^{2}\right)$ had a worse quality of $\chi^{2} / \mathrm{dof}=1.31$ with four fit parameters when applying the cuts $Q^{2}<0.3 \mathrm{GeV}^{2}$ and $m_{\pi}<400 \mathrm{MeV}$. However, we noticed that the $Q^{2}$ dependence of the SSE expression was in worse agreement than in the case of $F_{1}\left(Q^{2}\right)$, while the $m_{\pi}$ dependence was better. The resulting Pauli radii and anomalous magnetic moments are shown in Fig. 2. The left panel shows the Pauli radius $\left\langle r_{2}^{2}\right\rangle$, while the right panel shows the anomalous magnetic moment, $\kappa_{v}$. Again, data points from tripole fits are included in the plot, but have no influence on the fit. We find that $\left\langle r_{2}^{2}\right\rangle$ is described well by the fit and the fit even gets close
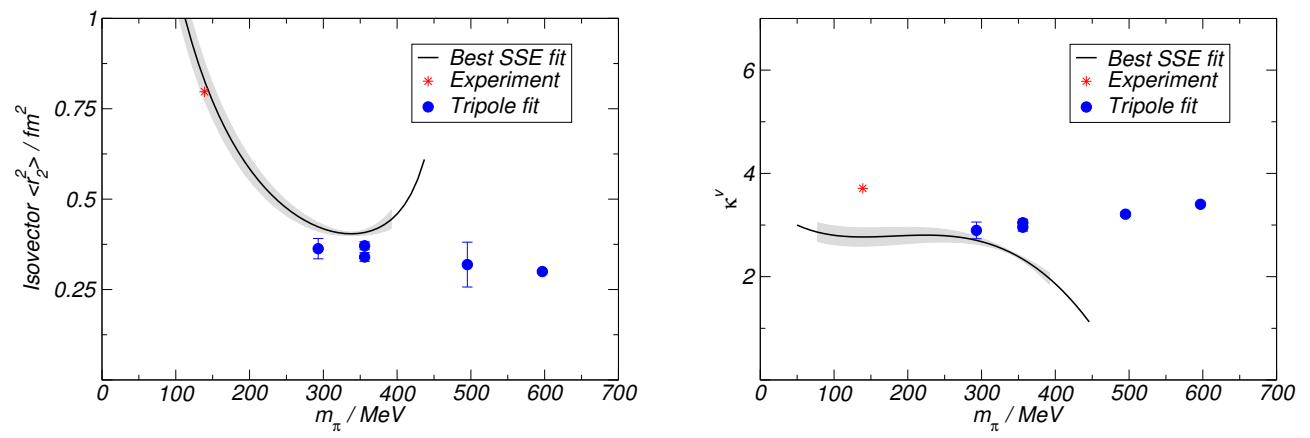

Figure 2: Isovector Pauli radius $\left\langle r_{2}^{2}\right\rangle$ (left panel) and anomalous magnetic moment $\kappa_{v}$ (right panel) as a function of the pion mass.

to the experimental point. The magnetic moment $\kappa_{v}$ underestimates the experiment. We find these fits encouraging, but believe that the excellent agreement with experiment is accidental for $\left\langle r_{2}^{2}\right\rangle$. 
Data with smaller pion mass is needed to verify this finding since $\kappa_{v}$ does not yet agree, although it originates from the same data set.

Similar to our fit strategy for the vector form factors, we also adopt a simultaneous fit to the $Q^{2}$ and $m_{\pi}$-dependence of the axial form factor, $G_{A}\left(Q^{2}\right)$. Figure 3 shows the result of the chiral fit together with the dipole fit and the data set for the $28^{3}$ lattice at $m_{\pi}=355.98 \mathrm{MeV}$ with a fitting range of $Q^{2}<0.4 \mathrm{GeV}^{2}$ for the SSE expansion and all $Q^{2}$ values for the dipole fit. The SSE fit gives a $\chi^{2} / \mathrm{dof}=1.73$. The resulting axial radius, however, is $\left\langle r_{A}^{2}\right\rangle=0.1560(60) \mathrm{fm}^{2}$ for the cuts $Q^{2}<0.4 \mathrm{GeV}^{2}$ and $m_{\pi}<400 \mathrm{MeV}$, substantially underestimating the experimental value from Ref. [22]. Future lattice calculations at smaller pion masses will be crucial to resolve this issue.

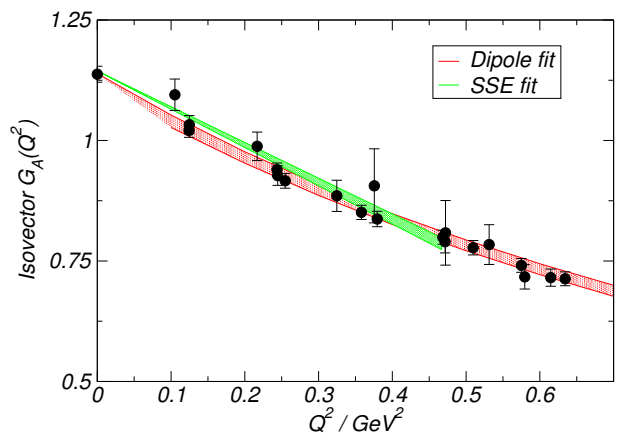

Figure 3: Comparison of dipole and chiral fit to $G_{A}\left(Q^{2}\right)$ for the $28^{3}$ lattice at $m_{\pi}=355.98 \mathrm{MeV}$.

The induced pseudoscalar form factor, $G_{P}\left(Q^{2}\right)$, is not described by a dipole type fit formula. Instead, it is commonly fit using a pion-pole expression, giving excellent agreement with the data, cf. Ref. [22]. We performed two kinds of fits: First, we repeated the analysis done previously with the other form factors, i.e. performing a combined fit in $Q^{2}$ and $m_{\pi}$. Second, we took the pion-pole form as a function of $Q^{2}$ and fit it using a single ensemble with fixed $m_{\pi}$, treating the pion mass as a free parameter.

In the first case, we again find that kinematic cuts of $Q^{2}<0.5 \mathrm{GeV}^{2}$ and $m_{\pi}<400 \mathrm{MeV}$ yields reasonable results. In the second case, we find the location of the pion pole, $m_{\pi}=417(43) \mathrm{MeV}$, with an uncertainty of $10 \%$ within the actual value of $m_{\pi}=355.98 \mathrm{MeV}$ on the $28^{3}$ lattice. The resulting $\chi^{2} / \mathrm{dof}=0.94$ indicates an excellent fit to the data. Figure 4 shows a comparison of the two fits. It is evident that both fits manage to describe the data well, but the uncertainty of the curve with $m_{\pi}$ as a free parameter is larger. We conclude that the isovector $G_{P}\left(Q^{2}\right)$ is described well by the pion-pole form

\section{Summary and Outlook}

We have computed the isovector form factors of the vector and the axial current for the nucleon within our framework of mixed action calculations. We have applied new analysis techniques that result in substantially reduced error bars at minimal additional computational cost. Furthermore, due to the agreement between the lattice results reported in this proceeding and the corresponding one reported in [8] obtained using full DWF, we are confident that the hybrid calculations employed do not suffer from systematic effects. 


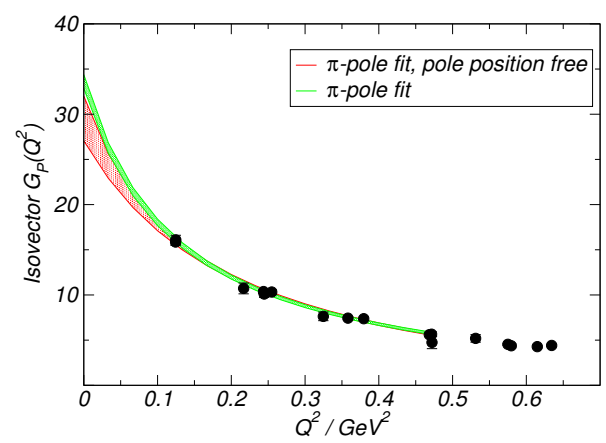

Figure 4: Comparison of pion-pole fits to isovector $G_{P}\left(Q^{2}\right)$ on the $28^{3}$ lattice with fixed pion pole and with the pion pole as a free parameter.

We find that a combination of chiral fits and lattice data is possible with the current generation of lattice calculations. This way, we obtain qualitative agreement with many features we expect to hold when approaching the chiral limit. We expect that the upcoming generation of lattice calculations will provide conclusive quantitative results from first principles that will be in agreement with experiment without resorting to assumptions on functional behavior outside of what can be predicted by chiral perturbation theory. We are able to provide fits to the vector form factors, $F_{1}\left(Q^{2}\right)$ and $F_{2}\left(Q^{2}\right)$. While we have no explanation for the discrepancy of the axial radius, $\left\langle r_{A}^{2}\right\rangle$, we find that induced pseudoscalar form factor is described well by the pion-pole form.

\section{Acknowledgments}

This work was supported in part by U.S. DOE Contract No. DE-AC05-06OR23177, by the DOE Office of Nuclear Physics under grants DE-FG02-94ER40818, DE-FG02-04ER41302, DEFG02-96ER40965, DE-FG02-05ER25681 and DE-AC02-06CH11357 and the EU (I3HP) under contract No. RII3-CT-2004-506078. Ph.H. and B.M. acknowledge support by the Emmy-Noether program and the cluster of excellence "Origin and Structure of the Universe" of the DFG and W.S. acknowledges support by the National Science Council of Taiwan under the grant numbers NSC96-2112-M002-020-MY3 and NSC96-2811-M002-026 and wishes to thank the Institute of Physics at Academia Sinica for their kind hospitality and support as well as Jiunn-Wei Chen at National Taiwan University and Hai-Yang Cheng and Hsiang-Nan Li at Academia Sinica for their hospitality and for valuable physics discussions and suggestions. K.O. acknowledges support from the Jeffress Memorial Trust grant J-813 and Ph.H., M.P. and W.S. acknowledge support by the A.v. Humboldt-foundation through the Feodor-Lynen program. This research used resources under the INCITE and ESP programs of the Argonne Leadership Computing Facility at Argonne National Laboratory, resources provided by the William and Mary Cyclades Cluster, and resources provided by the New Mexico Computing Applications Center (NMCAC) on Encanto. These calculations were performed using the Chroma software suite [23]. We are indebted to members of the MILC Collaboration for providing the dynamical quark configurations that made our full QCD calculations possible. 


\section{References}

[1] LHPC Collaboration, in preparation.

[2] C. Allton et al. [RBC-UKQCD Collaboration], Phys. Rev. D 78, 114509 (2008).

[3] Ph. Hägler et al. [LHPC Collaboration], Phys. Rev. D 77, 094502 (2008).

[4] A. Walker-Loud et al., arXiv:0806.4549 [hep-lat].

[5] D. B. Renner et al. [LHP Collaboration], Nucl. Phys. Proc. Suppl. 140, 255 (2005).

R. G. Edwards et al. [LHPC Collaboration], PoS LAT2005, 056 (2006).

W. Schroers, Nucl. Phys. A 755, 333 (2005).

J. W. Negele et al. [LHPC Collaboration], Int. J. Mod. Phys. A 21, 720 (2006).

R. G. Edwards et al. [LHPC Collaboration], Phys. Rev. Lett. 96, 052001 (2006).

W. Schroers, Eur. Phys. J. A 31, 784 (2007).

C. Alexandrou, G. Koutsou, H. Neff, J. W. Negele, W. Schroers and A. Tsapalis, Phys. Rev. D 77, 085012 (2008).

[6] S. N. Syritsyn et al., PoS LATTICE2008, 169 (2008).

[7] S. N. Syritsyn et al., arXiv:0907.4194 [hep-lat].

[8] M.F. Lin [LHPC Collaboration], these proceedings.

[9] M. Göckeler et al. [QCDSF Collaboration and UKQCD Collaboration], arXiv:0709.3370 [hep-lat]. M. Göckeler et al. [QCDSF/UKQCD Collaboration], PoS LAT2007, 161 (2007).

H. W. Lin, T. Blum, S. Ohta, S. Sasaki and T. Yamazaki, Phys. Rev. D 78, 014505 (2008).

[10] J. M. Zanotti, arXiv:0812.3845 [hep-lat].

[11] D.B. Renner, these proceedings.

[12] C. Aubin et al., Phys. Rev. D 70, 094505 (2004).

[13] T. Blum, C. Dawson and T. Izubuchi, private communication.

[14] A. Ali Khan et al. [CP-PACS Collaboration], Phys. Rev. D 65, 054505 (2002) [Erratum-ibid. D 67, 059901 (2003)].

[15] C. Amsler et al. [Particle Data Group], Phys. Lett. B 667, 1 (2008).

[16] G. Hohler, E. Pietarinen, I. Sabba Stefanescu, F. Borkowski, G. G. Simon, V. H. Walther and R. D. Wendling, Nucl. Phys. B 114, 505 (1976).

[17] P. Mergell, U. G. Meissner and D. Drechsel, Nucl. Phys. A 596, 367 (1996).

[18] M. A. Belushkin, H. W. Hammer and U. G. Meissner, Phys. Rev. C 75, 035202 (2007).

[19] J. Arrington, W. Melnitchouk and J. A. Tjon, Phys. Rev. C 76, 035205 (2007).

[20] V. Bernard, H. W. Fearing, T. R. Hemmert and U. G. Meissner, Nucl. Phys. A 635, 121 (1998) [Erratum-ibid. A 642, 563 (1998 NUPHA,A642,563-563.1998)].

[21] T. R. Hemmert and W. Weise, Eur. Phys. J. A 15, 487 (2002).

[22] V. Bernard, L. Elouadrhiri and U. G. Meissner, J. Phys. G 28, R1 (2002).

[23] R. G. Edwards and B. Joo [SciDAC Collaboration and LHPC Collaboration and UKQCD Collaboration], Nucl. Phys. Proc. Suppl. 140, 832 (2005). 